\title{
Colour Sorting Robot in LabVIEW Using Image Processing
}

\author{
H. R. Ramesh ${ }^{1}$, Pooja ${ }^{2}$ \\ ${ }^{1}$ Associate professor, Dept. of EE, UVCE, Bangalore, Karnataka, India \\ ${ }^{2}$ PG Student [C\&I], Dept. of EE, UVCE, Bangalore, Karnataka, India
}

\begin{abstract}
The robotic manipulator plays a dominant role in the domain of process industries based applications such as pick and drop, quality based sorting, etc. Now a day's various automation techniques are being adopted \& researched on for increase in productivity, for better accuracy, eliminating the human errors and for safety. Therefore, the efforts are made to design and implementation of automatic technique to determine colour of object using image processing technique.USB camera is used as a vision sensor to measure the dimensions of the object to be picked. The USB camera collects the image of the object is transferred to the LabVIEW with image processing toolkits and modules to process the image. The robotic arm is designed with servomotors. Digital image processing algorithms are implemented to process the image captured by the USB camera to find the exact dimension of the object. NI-IMAQ Machine vision based functions are implemented and the results are presented.
\end{abstract}

Keywords: Digital Image Processing, Colour Sorting, Colour recognition, Robot, LabVIEW

\section{Introduction}

Robot and automation is employed in order to replace human work to perform those tasks that are routine, dangerous, complex and in hazardous area. In a world of advanced technology today, automation greatly increases production capability; improve product quality and lower production cost. It takes just few people to program or monitor the computer and carry out routine maintenance. Advanced robotic technologies add convenience to human life, not only in industrial applications, but also in the fields of education and entertainment. Researchers have developed numerous types of robots to meet different demands, so that the human-robot interactions are simplified and robots can carry out more complex functions. There are many kinds of robotic systems, but the robot arm is the one most used. LABVIEW software is used as a tool to achieve the goal of this project. LABVIEW software is one of the most powerful software using worldwide and it also consist a lot of image processing library which can be used for object detection. For this project, the program code is written using this software and it shall be efficient to detect the objects, eliminate the background image and detects the object.

Colour sensing and colour sorting is tedious task in many packaging industries. The robotic arm is developed with three degree of freedom. In early days, manual sorting is the method used for this purpose. But it is time consuming, tedious, slow and variant process. It is useful to sort small sized object based on its property known as colour. It works well and sort the object. Mainly, it has two steps, colour sensing and colour sorting. Colour sensing is done by the image processing. And colour sorting is done by servo motors. These two steps are integrated by Arduino UNO.

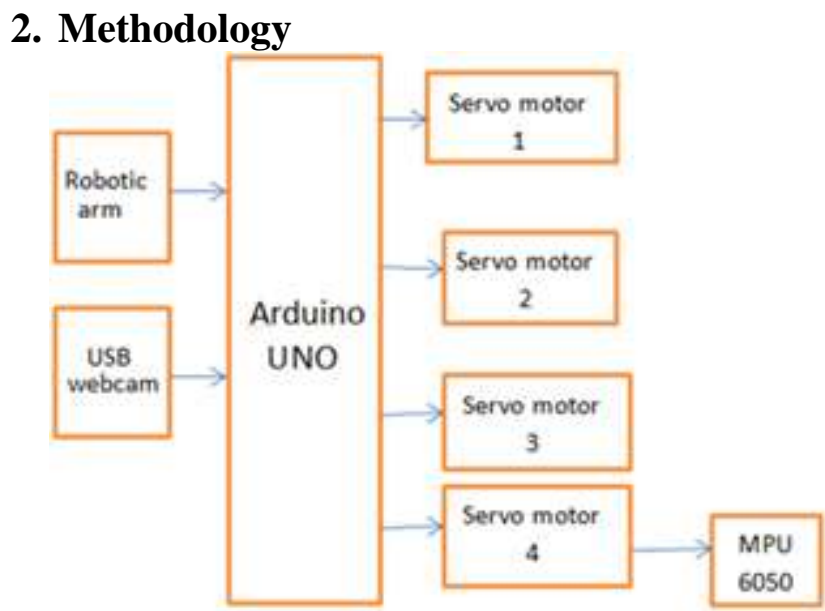

Figure 2.1: Block Diagram

This section deals with the explanation of the block diagram of the colour sorting robot using image processing as shown in fig 1. The controller used is an Arduino UNO which is interfaced to all the sensors and modules for getting the desired output. Pattern matching is used to detect the object. Pattern matching is a simple way of identifying the colour as well as shape of the object. In this method, first image is captured and then a part of the image to be identified and saved as a template in .png format. When the vision system runs, images are being captured and processed, the vision system searches for the template provided by the user. As soon as the template is found i.e., the pattern is matched; the sorting system takes the necessary action. Once the object is detected robotic arm move towards the object, pick the object depending on its shape and colour and it releases to the desired location.MPU6050 provides the tilt angle of the servo motor for the robotic arm to rotate. Even if the target position is changed, the robotic arm change to the new point. 


\section{Hardware Requirements}

\section{A. Camera}

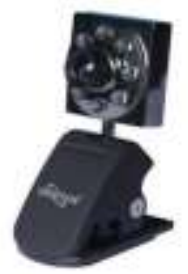

Figure 2.2: USB Webcam

The camera used in this case is tech com SSD-650 whose technical specifications are:

\section{Technical Specifications}

Image Sensor 1/7" CMOS sensor

Image Resolution 1280x960, 1024x1280, 1600x1200, 4032x2034

Focus distance $4 \mathrm{Cm} \sim$ infinity

Lens View angle 54 Degree

I/O Interface USB 1.1, 2.0

Power Consumption 160MW Typical

\section{B. Arduino UNO}

TheArduino Uno development board act as the communication link between the computer (LABVIEW) and the microcontroller through the COM port. The Arduino Uno is a microcontroller board based on the ATmega328. It has 14 digital input/output pins (of which 6 can be used as PWM outputs), 6 analog inputs, a $16 \mathrm{MHz}$ ceramic resonator, a USB connection, a power jack, an ICSP header, and a reset button. The Arduino Uno can be powered via the USB connection or with an external power supply. The board can operate on an external supply of 6 to 20 volts. If supplied with less than $7 \mathrm{~V}$, however, the $5 \mathrm{~V}$ pin may supply less than five volts and the board may be unstable. If using more than $12 \mathrm{~V}$, the voltage regulator may overheat and damage the board. The recommended range is 7 to 12 volts.

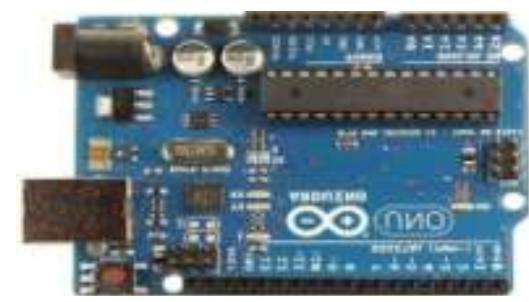

Figure 2.3: Arduino UNO

\section{Servo Motor}

Servos are DC motors with built in gearing and feedback control loop circuitry. And no motor drivers required. A servomotor is a rotary actuator that allows for precise control of angular position.

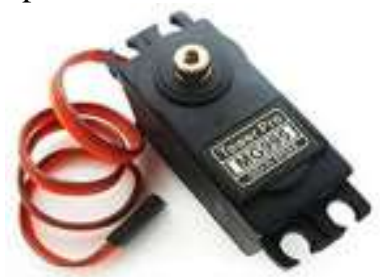

Figure 2.4: MG995 Servo motor
To fully understand how the servo works, we need to take a look under the hood. Inside there is a pretty simple set-up: a small DC motor, potentiometer, and a control circuit. The motor is attached by gears to the control wheel. As the motor rotates, the potentiometer's resistance changes, so the control circuit can precisely regulate how much movement there is and in which direction.

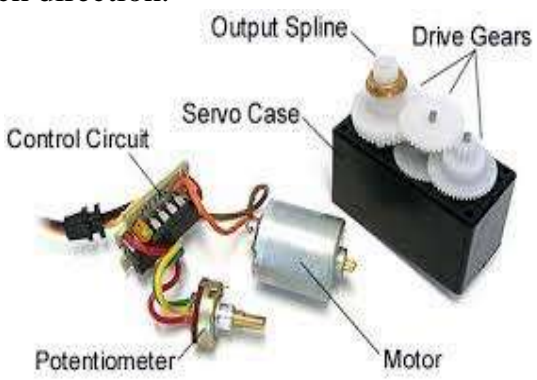

Figure 2.5: Servo motor parts

When the shaft of the motor is at the desired position, power supplied to the motor is stopped. If not, the motor is turned in the appropriate direction. The desired position is sent via electrical pulses through the signal wire. The motor's speed is proportional to the difference between its actual position and desired position. So, if the motor is near the desired position, it will turn slowly, otherwise it will turn fast. This is called proportional control. This means the motor will only run as hard as necessary to accomplish the task at hand.

Servos are controlled by sending a pulse of variable width, or pulse width modulation (PWM), through the control wire. There is a minimum pulse, a maximum pulse, and a repetition rate. A servo motor can usually only turn 90 degrees in either direction for a total of 180-degree movement. The motor's neutral position is defined as the position where the servo has the same amount of potential rotation in the both the clockwise or counter-clockwise direction. The PWM sent to the motor determines position of the shaft, and based on the duration of the pulse sent via the control wire; the rotor will turn to the desired position. The servo motor expects to see pulse every 20 milliseconds and the length of the pulse will determine how far the motor turns. For example, a $1.5 \mathrm{~ms}$ pulse will make the motor turn to the 90 -degree position. Shorter than $1.5 \mathrm{~ms}$ moves it to 0 degrees, and any longer than $1.5 \mathrm{~ms}$ will turn the servo to 180 degrees, as diagrammed below

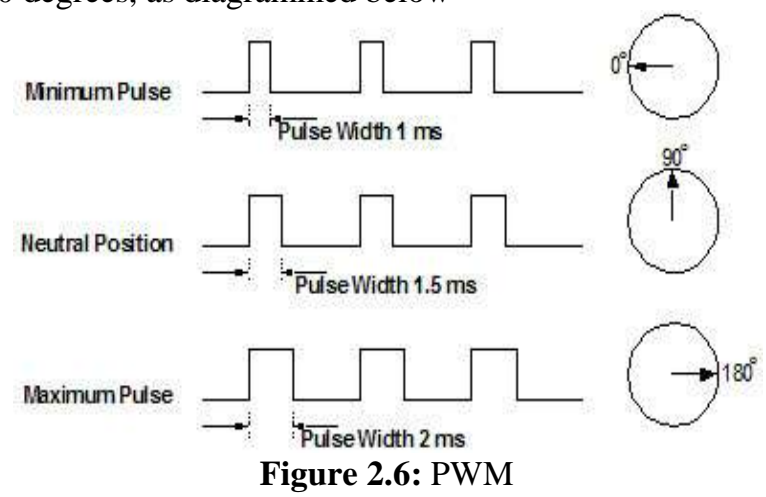

Servo Motor Control: The servo motor can be moved to a desired angular position by sending PWM (pulse width modulated) signals on the control wire. The servo understands the language of pulse position modulation. A

Volume 6 Issue 12, December 2017 


\section{International Journal of Science and Research (IJSR) \\ ISSN (Online): 2319-7064}

Index Copernicus Value (2016): 79.57 | Impact Factor (2015): 6.391

pulse of width varying from 1 millisecond to 2 milliseconds in a repeated time frame is sent to the servo for around 50 times in a second. The width of the pulse determines the angular position. For example, a pulse of 1 millisecond moves the servo towards $0^{\circ}$, while a 2 milliseconds wide pulse would take it to $180^{\circ}$. The pulse width for in between angular positions can be interpolated accordingly. Thus, a pulse of width 1.5 milliseconds will shift the servo to $90^{\circ}$. Power supply for Servo: The servo requires a DC supply of $4.8 \mathrm{~V}$ to $6 \mathrm{~V}$. For a specific servo, its voltage rating is given as one of its specification by the manufacturer. The DC supply can be given through a battery or a regulator. The battery voltage must be closer to the operating voltage of the servo. This will reduce the wastage of power as thermal radiation. A switched regulator can be used as the supply for better power efficiency.

\section{MPU6050}

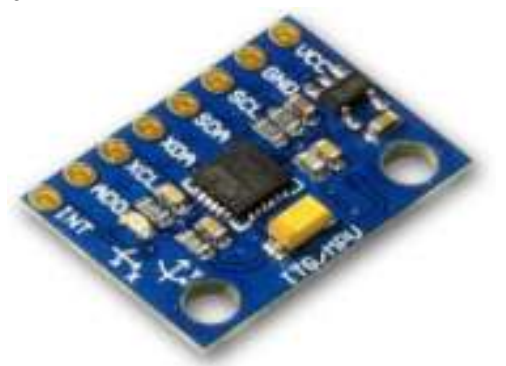

Figure 2.7: MPU 6050

The MPU6050 has an embedded 3-axis MEMS gyroscope, a 3-axis MEMS accelerometer. It is very useful for some motion detecting. This small module integrates the logic level converter circuit (makes it compatible with $3.3 \mathrm{~V}-5 \mathrm{~V}$ voltage level) together with the MPU6050 sensor.The MPU60X0 has an embedded 3-axis MEMS gyroscope, a 3-axis MEMS accelerometer, and a Digital Motion Processor (DMP) hardware accelerator engine with an auxiliary I2C port that interfaces to 3rd party digital sensors such as magnetometers. When connected to a 3-axis magnetometer, the MPU-60X0 delivers a complete 9-axis MotionFusion output to its primary $\mathrm{I} 2 \mathrm{C}$ or SPI port (SPI is available on MPU-6000 only). The MPU-60X0 combines acceleration and rotational motion plus heading information into a single data stream for the application.

\section{E. Voltage Regulator}

Voltage regulator is a device that is used to make a voltage constant/ linear. Here this is a 5 Volt regulator circuit diagram using IC LM7805. Voltage sources in a circuit may have fluctuations resulting in not giving fixed voltage outputs. Voltage regulator IC maintains the output voltage at a constant value. 7805 IC, a voltage regulator integrated circuit (IC) is a member of 78xx series of fixed linear voltage regulator ICs used to maintain such fluctuations. The $\mathrm{xx}$ in $78 \mathrm{xx}$ indicates the fixed output voltage it provides. 7805 IC provides +5 volts regulated power supply with provisions to add heat sink as well.

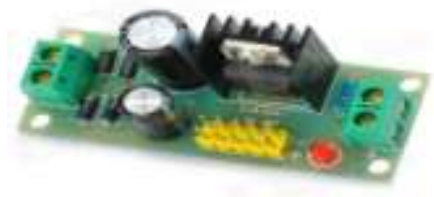

Figure 2.8: voltage regulator

\section{Software Requirements}

NI LABVIEW (Laboratory Virtual Instrumentation Engineering Workbench) is a graphical program (Gprogramming) development environment from National Instruments (www.ni.com). Programs written in LABVIEW are called VIs (virtual instruments). It is a powerful tool used by engineers and scientists for different kind of measurements, process controls and R\&D (Research and Development). It can easily be integrated with most of the hardware, provided the driver of the hardware is in-stalled into the computer. LABVIEW can also be integrated with other software tools such as Matlab and Simulink. It can be connected to different PLCs (Programmable Logical Controllers) via different industrial communication protocols. One advantage of programming in LABVIEW is that we don't have the overhead to write huge codes.

\section{Implementation}

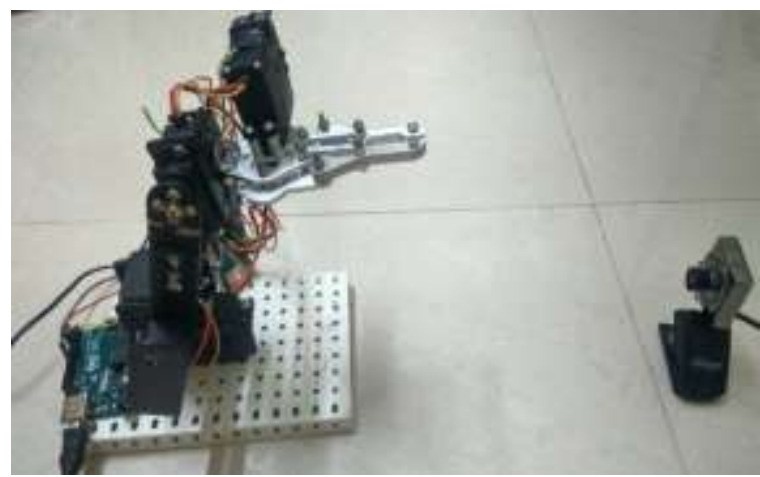

Figure 3.1: Prototype model of colour sorting robot

Figure shows the hardware setup of the colour sorting robot using image processing. The hardware setup consists of robotic arm and webcam. The camera is placed in front of the robotic arm. The robotic arm with three degree of freedom is developed. First the camera is initialized, as soon as the object is detected based on its shape and colour. The robotic arm move towards to the object. Gripper will hold the object and release the object in the desired location. Even if the desired location is changed, the robotic arm moves to the new point location and release the object. Similarly different objects are sorted and placed at the desired position. 


\section{International Journal of Science and Research (IJSR) \\ ISSN (Online): 2319-7064}

Index Copernicus Value (2016): 79.57 | Impact Factor (2015): 6.391

\section{Results and Discussion}

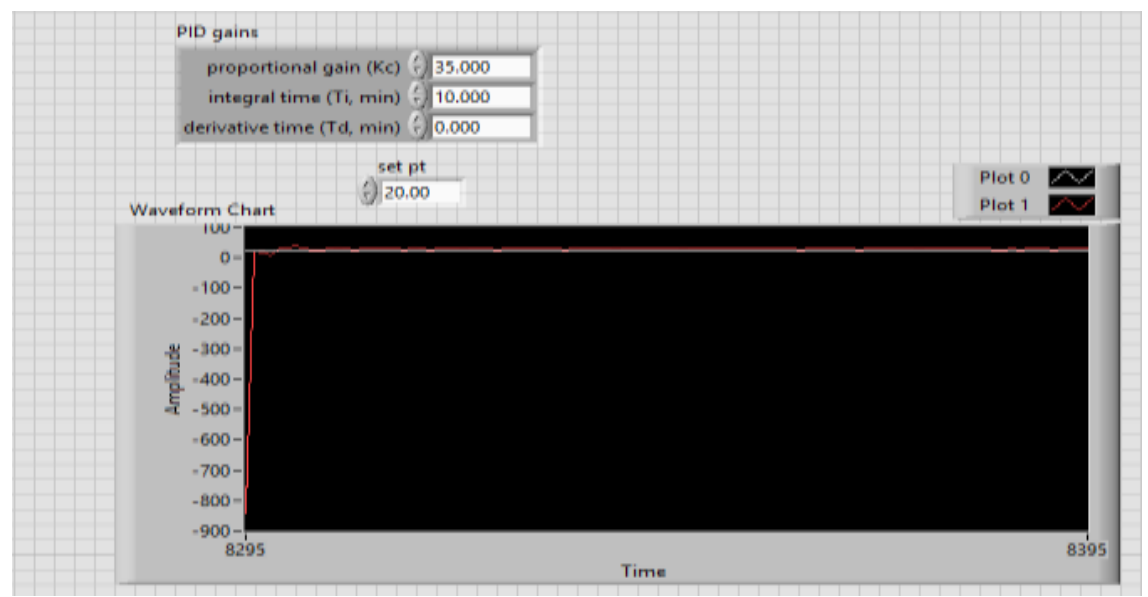

Figure 4.1: PID graph at set point 20 degree

The PID parameters are tuned in such a way that the servo motor is kept at a set point of 20degree. Now by trial and error method the process variable is continuously tuned to reach the set point under a shortest interval of time.Hence by trial and error method the PID parameters are obtained as follows

Table 1: Tuned PID parameters at set point 20 degree

\begin{tabular}{|c|c|}
\hline $\mathrm{Kp}$ & 35 \\
\hline $\mathrm{Ki}$ & 10 \\
\hline $\mathrm{Kd}$ & 0 \\
\hline
\end{tabular}

\section{Conclusion and Future Scope}

The purpose of our project is to detect the object by detecting colour as well as shape of the object. Segmentation of object is done by using the edge detection, colour processing, and feature extraction. Colour processing eliminates the unrelated colour or the background image. The project shows the percentage of each primary colour and depending upon that percentage the colour of the particular object is detected. After implementing the image processing algorithms, thecolour detection and shape detection algorithm gave accurate results almost every time. However dark shadow of theobject around it can lead to wrong measurements in case of shape detection which was overcome by adoption of flashlight placed exactly above the object next to the camera. This helped to get a clear picture. Improvement can be made is to allow the robot to move freely in all direction. This can be carried out with the additional motion sensor that allow robot to detect object and move freely. The other improvement that can be made is allowing the robot to carry heavier object. This can be done by increasing the strength of the robot itself.

\section{References}

[1] RajinderTiwari "Design and Implementation of An Intelligent Robotic Manipulator Using Labview For industrial applications". Journal of Communications Technology, Electronics and Computer Science, 2016 ISSN 2457-905X, pp.7-14

[2] Ramish, SsyedBaqarHussain"Design of A 3 DOF Robotic Arm" International conference 2016, pp.145-149
[3] ShubhangiWanve, B.G. Gawalwad "Automatic colour object sorting system" SInternational Journal of Modern Trends in Engineering and Research, e-ISSN No.:23499745, Date: 2-4 July, 2015,pp.1423-1429

[4] Nisha, Dinesh kumar, Sekar and Indira "Vision Assisted Pick and Place Robotic Arm "Advances in Vision Computing: An International Journal (AVC) Vol.2, No.3, September 2015, pp.9-18

[5] Jih-gauJuang, Yi-ju Tsai and Yang-wuFan "Visual Recognition and its Application to Robot Arm control"'ISSN 2076-3417 www.mdpi.com/journal/applsci 2015 , pp.851-880

[6] http://home.hit.no/ hansha "Introduction to Vision Systems in LabVIEW" 